\title{
Coordinación docente y precariedad. Modelos de coordinación en la enseñanza de la Sociología en Andalucía
}

\author{
Coordination of Teachers and Precariousness. Coordination Models in the \\ Teaching of Sociology in Andalusia
}

José Manuel Torrado, Henar Baldán y Ricardo Duque-Calvache'

\begin{abstract}
Resumen
Desde la implantación de los Sistemas de Garantía de Calidad, la enseñanza universitaria ha incorporado mecanismos y figuras de coordinación a la práctica docente. Sin embargo, su aplicación dista de ser homogénea. Un mecanismo diseñado originalmente para la mejora de la calidad docente ha cobrado una nueva significación e importancia debido al contexto de precarización generalizada de las plantillas de profesorado en la universidad. Este trabajo plantea un análisis del contexto, las bases teóricas y los distintos modelos de coordinación implementados en una serie de universidades donde se imparte el grado de Sociología en Andalucía, pero los resultados son perfectamente extrapolables a otras disciplinas y lugares. Empleando un análisis autoetnográfico, a partir de la experiencia de los autores del texto, se elaboran dos modelos típico-ideales de coordinación. Las prácticas docentes reales en los diferentes departamentos analizados se mueven en un continuo que va desde la coordinación total a la autonomía total. Cada uno de estos modelos tiene una serie de ventajas e inconvenientes, y son fruto de una particular combinación de factores materiales, culturales y humanos. Nuestros resultados muestran la utilidad de un mayor grado de coordinación en contextos de plantillas inestables o en formación, frente a la adecuación de la autonomía a las situaciones de mayor estabilidad y experiencia. La coordinación puede servir para mitigar algunos problemas causados por la precariedad, pero no debería ser una coartada para profundizar en ella.
\end{abstract}

\section{Palabras clave}

Coordinación docente, precariedad, docencia universitaria, sociología.

\section{Abstract}

Since the implementation of the Quality Assurance Systems, university education has incorporated coordination mechanisms and figures into teaching practice. However, their application is far from being homogeneous. A mechanism originally designed to improve teaching quality has taken on new significance and importance due to the context of generalized precariousness of university academic staff. This paper presents an analysis of the context, the theoretical bases and the different coordination models implemented of a series of universities where the Sociology degree is taught in Andalusia, but the results are perfectly extrapolated to other disciplines and places. Through the use of the autoethnographic analysis, based on the experience of the authors of the text, two typical ideal models of coordination are elaborated. The actual teaching practices in the different departments analysed move along a continuum ranging from total coordination to total autonomy. Each of these models has a number of advantages and disadvantages, and is the result of a particular combination of material, cultural and human factors. Results show the usefulness of a higher degree of coordination in contexts of unstable or training workforces, as opposed to the appropriateness of autonomy in situations of greater stability and experience. Coordination may serve to mitigate some problems caused by precariousness, but it should not be an alibi for deepening it.

Keywords

Teaching coordination, precariousness, university teaching, sociology.

\section{Cómo citar/Citation}

Torrado, José Manuel; Baldán, Henar y Duque-Calvache, Ricardo (2021). Coordinación docente y precariedad. Modelos de coordinación en la enseñanza de la Sociología en Andalucía. Revista de Sociología de la Educación-RASE, 14 (3), 370-390. http://dx.doi.org/10.7203/ RASE.14.3.20902. 


\section{Introducción}

En un entorno laboral crecientemente precarizado, las universidades cuentan con plantillas aquejadas de un alto porcentaje de temporalidad y sometidas a una frecuente rotación (Marugán-Pintos y Cruces-Aguilera, 2013; Santos-Ortega, Muñoz-Rodríguez y Poveda-Rosa, 2015). El currículum del personal docente en sus fases iniciales suele reflejar este fenómeno, y es habitual encontrar vidas laborales compuestas de una sucesión de contratos breves con diversas universidades (Agulló-Fernández, 2013; Díaz-Santiago, 2013) hasta que —en el mejor de los casos — se produce la estabilización laboral, en periodos ya bastante avanzados de la carrera profesional (Castillo y Moré, 2016). Estas condiciones laborales, que son compartidas en buena parte en todas las universidades españolas, han alterado de manera sustancial la importancia de la coordinación a diferentes niveles. En el pasado, y con un entorno laboral más estable, la coordinación podía ser vista como una interferencia en la libertad de cátedra; en un contexto de altísima precariedad y rotación, la coordinación se erige como una necesidad de primer orden.

Desde un punto de vista normativo, la coordinación entre docentes de una misma asignatura es una cuestión de vital importancia, no sólo porque contribuye a la construcción colaborativa de metodologías y contenidos, sino porque garantiza la igualdad de oportunidades y trato de los estudiantes en el proceso de enseñanza-aprendizaje (Ortiz-Oria, et al., 2011; Ruiz y Torrego-Egido, 2011). No obstante, desde una perspectiva materialista, la coordinación es una necesidad, impuesta por el contexto de precariedad. La creciente rotación de personal docente se traduce en constantes entradas y salidas de profesorado, incluso durante un mismo curso, siendo cada vez más común que la persona que inicia el curso no sea la misma que lo termine. Esta fragmentación e itinerancia se traslada necesariamente a la realidad del aula, despertando la necesidad de coordinar contenidos, materiales y métodos pedagógicos a fin de minimizar los efectos negativos de la precariedad sobre el proceso de aprendizaje.

Si bien la coordinación entre asignaturas, y la coordinación en general, se da por supuesta desde la introducción del popularmente llamado Plan Bolonia, la realidad dista mucho de lo que las normas reflejan (Bolarin-Martínez y Moreno-Yus, 2015). Cada universidad, cada departamento, ha concretado estas normas de manera diferente, pero incluso más diversa es la forma de aplicación y su concreción en lo que aquí llamaremos «modelos de coordinación docente». Más allá de las figuras normativas que regulan la coordinación, estos modelos dependen de otros factores o determinantes que hacen que la aplicación de tales normas sea variable, diferente, e incluso casi anulada. Nos referimos a aspectos como la cultura organizativa - las creencias, valores, formas de pensar y hacer del profesorado- y las condiciones materiales que se dan en cada departamento.

En el presente trabajo partimos del análisis autoetnográfico de la enseñanza de asignaturas de Sociología en diferentes universidades andaluzas para plantear un análisis de los distintos modelos de coordinación entre asignaturas de forma típico-ideal, puesto que consideramos que las condiciones y circunstancias son muy similares en otras universidades y disciplinas. Para ello, en primer lugar, analizamos el actual contexto universitario, caracterizado por una creciente precarización del profesorado universitario que va en paralelo a una cada vez mayor implantación de figuras de coordinación. En segundo lugar, se propone un marco teórico para entender la coordinación de asignaturas, profundizando en dos aspectos fundamentales. Primero, en los factores determinantes que, en cada departamento, hacen concretarse unos modelos de coordinación docente u otros (la estructura normativa, la cultura organizativa y las condiciones materiales). Segundo, en las dimensiones o ítems fundamentales en los que dichos modelos se 
concretan. Tras esta exposición más teórica, se plantea una reflexión, a partir de la experiencia laboral y observación que los y las autoras han podido hacer acerca de la realidad de la coordinación docente en los departamentos donde han trabajado. En concreto, los departamentos de Sociología de la Universidad de Huelva, la Universidad Pablo de Olavide de Sevilla, la Universidad de Sevilla y la Universidad de Granada. A partir de esta vivencia de los distintos modelos de coordinación en las mencionadas universidades, se plantea un continuo teórico estructurado en torno a dos polos, dos modelos típico-ideales de coordinación docente: la coordinación total y la autonomía total. Creemos que este continuo permite abrir una necesaria reflexión sobre los problemas, ventajas, debilidades y oportunidades de los distintos modelos de coordinación docente realmente existentes y supone un primer paso hacia un repensar más sistemático de la realidad de la coordinación y su vinculación con la precariedad laboral.

\section{Precariedad y coordinación. Dos caras de una misma moneda}

Desde los inicios del presente siglo han confluido dos tendencias paralelas y aparentemente contradictorias en las universidades españolas. Por un lado, la progresiva implantación del llamado Espacio Europeo de Educación Superior (EEES) ha supuesto un proceso de transformación radical de las universidades, orientado según su diseño a lograr criterios de calidad y excelencia en la investigación y la docencia (Conesa-Carpintero y González-Ramos, 2018), siendo un pilar indispensable para esta meta la consolidación de las figuras de coordinación docente (Bolarin-Martínez, 2016). Por otro lado, el contexto de crisis económica de la pasada década, junto a las políticas de austeridad propuestas para combatirla, han supuesto un recorte sustancial del gasto público en universidades e investigación, con la consiguiente precarización del profesorado universitario (Castillo-Olivares, et al., 2020). Veamos con detalle estas dos tendencias.

La adaptación al EEES es un proceso relativamente rápido, que sienta sus bases en la reforma del sistema universitario propuesta en la Ley Orgánica de Universidades de 2001 (Ley Orgánica, 6/2001) y su posterior modificación en 2007 (Ley Orgánica, 4/2007). Sobre estos pilares normativos, se plantea una reconversión integral del sistema universitario español que fija como metas la calidad y excelencia, amén de una mayor conexión de la universidad con la sociedad y, sobre todo, con el ámbito privado. Es en este contexto en el que se asienta la llamada «cultura de la evaluación», materializada en hitos como la constitución de la ANECA en 2002 o la creación de rankings de universidades (Conesa-Carpintero y González-Ramos, 2018; Santos-Ortega, et al., 2015). Este conjunto de reformas, no se hicieron en un contexto pacífico y consensuado, nada más lejos, dio lugar a una oleada de movilización social, primero contra la LOU de 2001 (León-Sancho, 2008) y posteriormente contra la implantación del EEES (Fernández-González, 2014), reavivando un movimiento estudiantil hasta entonces poco activo. Entre las críticas a las reformas mencionadas, destacan especialmente la excesiva focalización de la universidad hacia el mundo empresarial, lo que algunas personas interpretaron como una privatización encubierta (Díez-Gutiérrez, 2009). Tampoco quedó exenta de críticas la llamada «cultura de la evaluación y la calidad», la cual ha llegado a ser interpretada como un incremento de la explotación laboral del profesorado universitario (Gill, 2014; Saura y Bolívar, 2019), a través de la imposición de unos métodos de trabajo propiamente tayloristas (Noll, 2019). Estas reformas no sólo afectaron al ámbito investigador, sino que tuvieron su efecto en la docencia. A través del Real Decreto 1393/2007 y su posterior modificación en 2010 (Real Decreto 861/2010) se estructura el nuevo sistema de enseñanza reglada universitaria de grado y posgrado, bajo la misma filosofía de la calidad, la excelencia y la adaptación a las demandas sociales y empresariales. Para cumplir con estos requerimientos, en el seno de las universidades se desarrollan los llamados 
Sistemas de Garantía de Calidad (SGC), los cuales tenían como fin el control, supervisión, revisión y mejora constante de las enseñanzas de grado y posgrado. Es en el contexto de estos SGC donde cobra importancia la figura de la coordinación a distintos niveles (Bolarin-Martínez, 2016). De esta manera, en las universidades españolas comienzan a implantarse distintas figuras de coordinación docente, la cuales, pese a ciertas variaciones contextuales, pueden clasificarse en tres tipos fundamentales: la coordinación de titulaciones, la coordinación de curso y la coordinación de asignaturas. Esta última, que tiene como fin garantizar unos criterios mínimos de calidad y homogeneidad en la impartición de una misma materia por parte del personal docente, es a la que dedicamos el presente trabajo ${ }^{2}$.

Sin embargo, esta apuesta reformista se ha desarrollado en un contexto de crisis y recortes, aparentemente contradictorio con los objetivos de calidad y excelencia docente perseguidos explícitamente por la legislación citada. Las políticas de austeridad que se implementaron para dar respuesta a la crisis económico-financiera de 2008, tuvieron un gran impacto en la universidad española, la cual vio reducida de manera drástica su presupuesto, pasando de un 2,26\% del gasto público total en 2008 a un 1,88\% en 2019 (Ministerio de Universidades y Ministerio de Educación y Formación Profesional, 2021a). Esta política de recortes ha tenido como traducción directa la mayor dificultad para conseguir financiación para proyectos de investigación (enfocando la misma a la captación de fondos privados y europeos), las reducciones de plantilla (vía despidos o mediante la reducción de las tasas de reposición) y una mayor precarización de las figuras contractuales del personal de administración (incrementándose las subcontratas) y del personal docente (Castillo y Moré, 2016; Conesa-Carpintero y González-Ramos, 2018). Sobre esto último, solo hace falta echar un breve vistazo a las estadísticas del personal universitario que facilita el actual Ministerio de Universidades para apreciar las consecuencias de los recortes en las plantillas docentes. En la tabla I, se muestra la evolución en términos porcentuales de las distintas figuras docentes en el sistema universitario español en el periodo 2015-2020. Esta evolución muestra una tendencia a la polarización de las plantillas, consolidándose un mercado de trabajo dual entre el personal docente. Por un lado, la evolución de las figuras de catedráticos y catedráticas y titulares da cuenta del retroceso de un mercado de trabajo primario caracterizado por buenas condiciones laborales y salarios elevados. Si bien las figuras de catedrático/a han subido en términos globales, las de titular han retrocedido casi en la misma medida, mostrando un sistema de promoción por la cúspide que no ha supuesto la consolidación como personal funcionario de muchas personas con la figura de contratado/a doctor/a. Por otro lado, si nos fijamos en las figuras de ayudante doctor/a, sustituto/a y asociado/a, se aprecia un ensanchamiento y consolidación de un mercado de trabajo secundario, caracterizado por la temporalidad y los bajos salarios. Especialmente relevante es la evolución del personal interino, cuyo gran incremento parece apuntar a que muchas universidades utilizan esta figura, a priori concebida para contingencias, como una solución a la carencia estructural de profesorado. La situación es aún peor si tenemos en cuenta que el porcentaje promedio de docentes a tiempo parcial en el curso 2019-2020 rondaba el 42\% (Ministerio de Universidades y Ministerio de Educación y Formación Profesional, 2021b).

2 El debate acerca de la conveniencia y éxito de la reforma, que merece sin duda ser abordado, no tiene cabida en este texto. Las figuras de coordinación y los mecanismos de control que establecen forman ya parte de la realidad universitaria. 
Tabla I. Evolución en términos porcentuales de las distintas figuras de profesorado universitario entre los cursos 2015-2016 y 2019-2020, por cursos y para todo el periodo

\begin{tabular}{llllll}
\hline & \multicolumn{1}{c}{} & & DIFERENCIA \\
\hline & $2016-2017$ & $2017-2018$ & $2018-2019$ & $2019-2020$ & $2015-2020$ \\
\hline Catedrático/a & $0,9 \%$ & $6,3 \%$ & $4,2 \%$ & $3,3 \%$ & $15,4 \%$ \\
\hline Titular & $-3,5 \%$ & $-3,6 \%$ & $-3,2 \%$ & $-1,2 \%$ & $-11,0 \%$ \\
\hline Contratado/a Doctor/a & $9,0 \%$ & $-0,5 \%$ & $-3,3 \%$ & $-2,1 \%$ & $2,6 \%$ \\
\hline Under Ayudante Doctor/a graduate & $1,5 \%$ & $15,1 \%$ & $22,3 \%$ & $18,5 \%$ & $69,3 \%$ \\
\hline Sustituto/a & $6,3 \%$ & $13,7 \%$ & $11,7 \%$ & $9,0 \%$ & $47,3 \%$ \\
\hline Asociado/a & $4,9 \%$ & $3,9 \%$ & $3,6 \%$ & $2,7 \%$ & $15,9 \%$ \\
\hline Otros contratos & $-6,3 \%$ & $-5,9 \%$ & $-4,7 \%$ & $0,1 \%$ & $-15,9 \%$ \\
\hline Total & $1,3 \%$ & $1,3 \%$ & $1,3 \%$ & $1,9 \%$ & $6,0 \%$ \\
\hline
\end{tabular}

Fuente: estadísticas del personal universitario. Ministerio de Universidades y Ministerio de Educación y Formación Profesional, 2021.

Esta situación de creciente precariedad tiene como correlato el retraso de la promoción entre el profesorado, produciéndose el coloquialmente llamado «tapón» en el acceso al mercado de trabajo primario. En el gráfico 1 se puede apreciar cómo el porcentaje que representan los mayores de cuarenta años se ha incrementado en las tres figuras docentes más precarias, reflejando que cada vez es más prolongada la permanencia en estas figuras que generalmente se venían considerando «de transición».

\section{Gráfico 1. Perfil por grupos de edad del profesorado ayudante doctor (PAD), asociado (PA) y sustituto interino (PSI) en el curso 2015-2016 y 2019-2020}

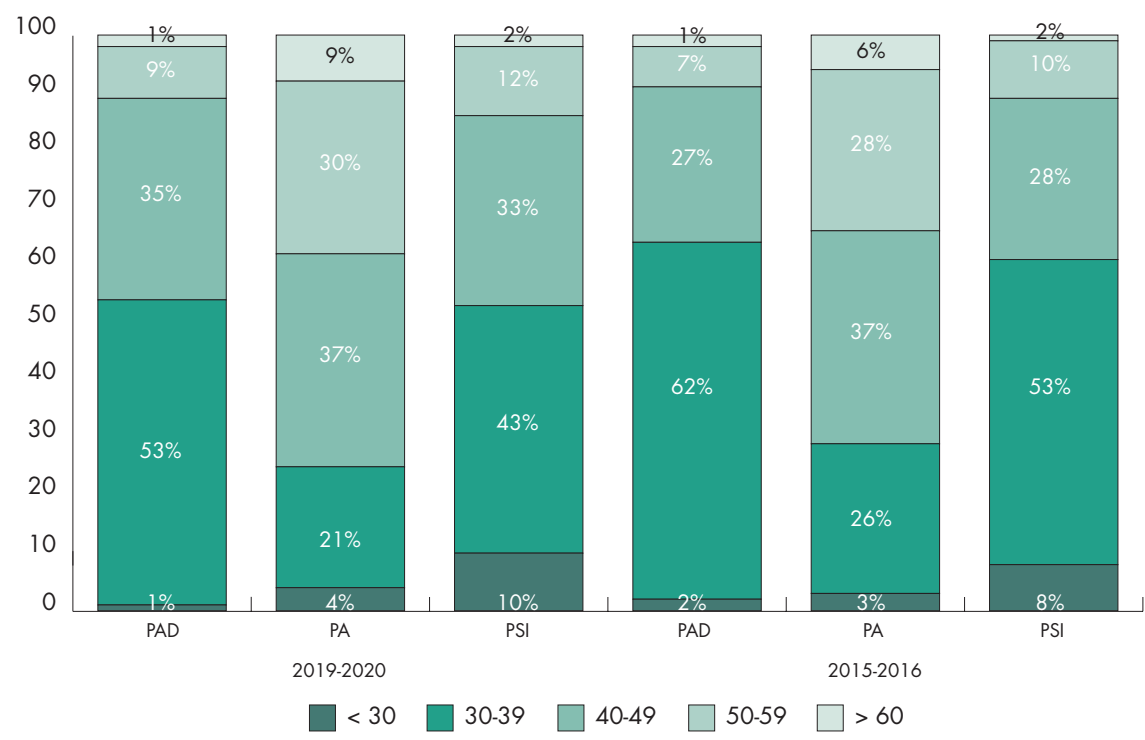

Fuente: estadísticas del personal universitario. Ministerio de Universidades y Ministerio de Educación y Formación Profesional, 2021.

Descritas ambas tendencias cabe preguntarse ¿es contradictorio el proceso de precarización docente con las reformas tendentes hacia una mayor calidad y coordinación en la docencia? Creemos que no. Lejos de ser tendencias contradictorias, la coordinación es una respuesta lógica a la consolidación de un mercado de trabajo docente polarizado y precarizado. La necesidad de contar con cada vez más personal itinerante y con bajos salarios supone que las universidades, facultades y departamentos tengan que plantear formas de garantizar que esta fragmentación y rotación en las plantillas docentes no se traduzca 
en una creciente fragmentación e inestabilidad en la impartición de la docencia. De esta manera, no es extraño que el proceso de precarización coincida en el tiempo con la implantación de los SGC en los que se sustentan los modelos de coordinación docente. Además, la creciente dualización del mercado de trabajo docente, no solo impone la necesidad de la coordinación, sino que crea su condición de posibilidad, al establecer una jerarquía laboral entre profesorado «senior» y precario, que permite (y en parte legitima) que los primeros estructuren un modelo docente que les es impuesto, con poca o ninguna negociación, a los segundos, aunque esto dependerá de muchos otros factores como veremos a continuación.

\section{Más allá de la precariedad. Una propuesta de marco teórico para entender la coordinación docente}

El contexto laboral precario descrito, junto al nuevo andamiaje normativo, consagraron la cristalización de las figuras de coordinación y la atribución de un creciente número de funciones a dichas figuras. Establecer una taxonomía de los modelos de coordinación docente requiere, en primer lugar, del desarrollo de un ejercicio deductivo, es decir, de la construcción de un marco teórico de referencia que sirva de guía para contrastar la realidad de la coordinación docente entre asignaturas en cada universidad, en cada departamento. Dos son las preguntas clave a las que deben darse respuesta para acometer tal tarea ¿Qué factores o determinantes inciden en la concreción de un modelo de coordinación docente en un departamento? ¿Qué características definen a un modelo de coordinación docente?

\subsection{Factores determinantes que inciden en la concreción de un modelo de coordi- nación docente}

Partiendo del análisis de Bolarin-Martínez (2016), pero ampliándolo con nuestras propias deducciones, creemos que tres son los factores determinantes de la concreción de un modelo de coordinación docente en un departamento (Gráfico 2). En primer lugar, la estructura normativa, entendiendo por esta el conjunto de normas formales — escritas - que definen cómo debe ser la coordinación y qué funciones debe cumplir. En segundo lugar, la cultura organizativa, definida como el conjunto de creencias, valores y formas de pensar y hacer docencia, de los miembros del departamento. En tanto que cultura, esta va más allá de las creencias y prácticas docentes propias de cada profesor/a, son un sistema cultural propio, con su propia estructura y que, como todo hecho social, está ahí antes de que entráramos al departamento y, posiblemente, seguirán estando allí cuando nos vayamos. Por último, pero no por ello menos importante, las condiciones materiales, entendiendo por estas el conjunto de condicionamientos sociales y materiales con los que tiene que lidiar el departamento: número de profesores/as, carga docente, tasa de rotación del profesorado, etc. La conjunción de estos tres factores (los cuales están también lógicamente relacionados entre sî), es lo que determina la concreción de un modelo de coordinación docente. 


\section{Gráfico 2. Los determinantes de los modelos de coordinación docente}

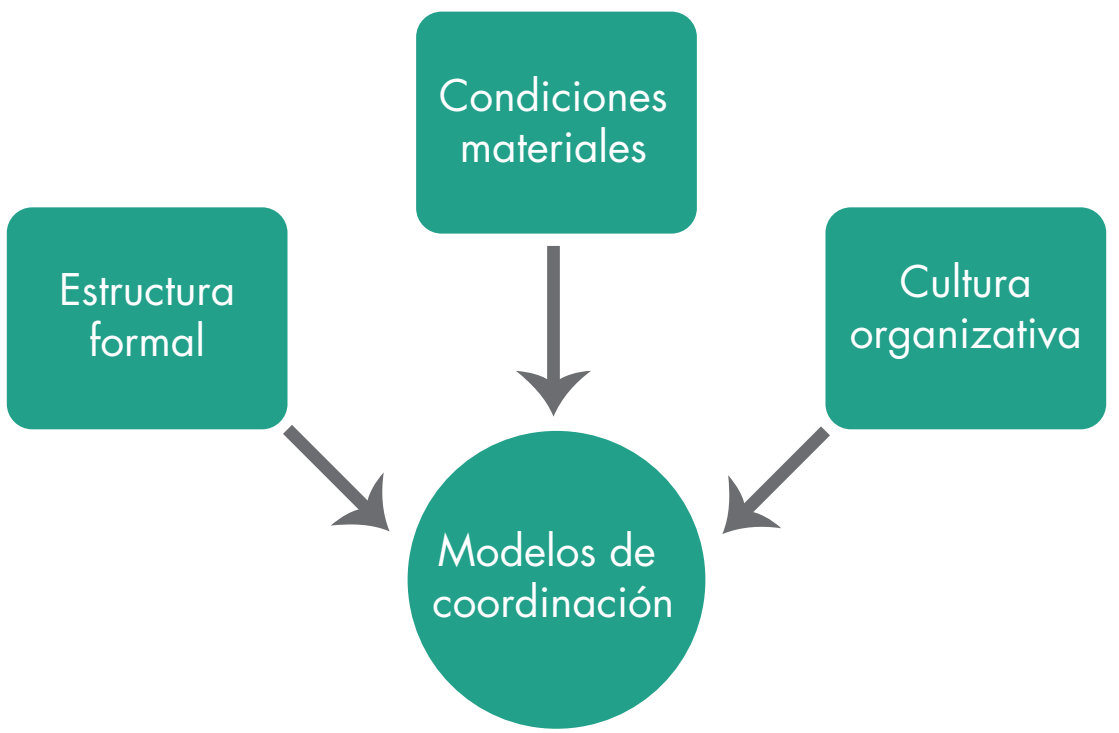

Fuente: elaboración propia.

Sobre el primer factor determinante, la estructura normativa, hace referencia a cómo está organizada la universidad. Constituye, por así decirlo, el andamiaje o esqueleto normativo que articula el funcionamiento institucional, y que ya se ha descrito someramente en el apartado anterior. No obstante, pese a que el marco normativo es común, y la mayoría de las universidades lo han incorporado, aunque de manera heterogénea (Bolarin-Martínez y Moreno-Yus, 2015), un elemento fundamental que condiciona los modelos de coordinación docente no es tanto la estructura normativa, como la cultura organizativa. Las normas, en tanto que imperativos burocráticos de obligado cumplimiento, están sujetas a interpretaciones en su ejecución, y los procedimientos por los cuales estas normas se llevan a cabo (se ejecutan) dependen de las formas de pensar y hacer del grupo humano que las practica, existiendo siempre cierto margen para cumplir la norma, pero dejándola sin efecto. De este modo, somos capaces de cumplir las normas escritas de manera ritualista, pero eludiendo su finalidad última y adaptándolas a nuestras creencias, demandas e intereses profesionales. De esto nos avisaba ya el teórico de la Sociología del Trabajo Michael Burawoy (1989), cuando nos hablaba del «juego de arreglárselas» que practicaban los trabajadores de la industria pesada, y cómo cumplían las normas de los patronos, pero adaptándolas a sus intereses, haciendo incluso que, por medio de arreglos informales, las normas se cumplieran, pero no siempre se consiguieran los objetivos que la patronal perseguía.

Reconectando con nuestro tema planteamos la pregunta ¿qué cultura organizativa impera en los departamentos universitarios de Sociología? ¿Qué consecuencias tienen estas para la concreción de modelos de coordinación docente? Son preguntas que no tienen respuesta sin un estudio previo. No obstante, parece que sí podemos elucidar de dónde venimos y hacia dónde parece que estamos yendo. Venimos de un modelo del docente «profesional», basado en los principios de competencia, vocación, licencia, independencia y autorregulación de los que hablaba Fernández-Enguita (1990). Un modelo basado en un desarrollo de la labor docente altamente autónoma pero también ciertamente aislada e individualista, que hace que el profesorado se comunique poco con sus compañeros/as y que ejecute sus propios métodos de enseñanza 
de manera individual. Este modelo del docente «profesional» ha sido, y en gran parte es, el que ha imperado en los departamentos de Sociología, y de todas las disciplinas, en las universidades españolas (Tomàs, et al., 2001). Por tanto, ha sido el tipo ideal que ha estructurado las creencias, las formas de hacer y pensar de los departamentos, en otras palabras: su cultura organizativa. Sin embargo, desde la llegada de la gran reforma educativa que supuso Bolonia, y los cambios normativos que implicó, este modelo se ve cada vez más cuestionado, especialmente en sus aspectos más negativos. La creación de las mencionadas figuras de coordinación supone un repensar de la propia cultura organizativa de los centros, y un revulsivo para su transformación hacia modelos de profesorado «profesional» pero «coordinado». Sin embargo, todavía estamos lejos de construir esa cultura organizativa que favorezca la construcción de auténticos modelos de coordinación (Bolarin-Martínez, 2016), aunque estamos en camino. El profesorado muestra resistencias a los modelos de coordinación. Resistencias basadas a veces en la competencia y el individualismo que este tipo cultural fomenta. Pero otras veces muy justificadas, y que descansan en los riesgos que implica la asunción de modelos de coordinación muy detallada para la autonomía del docente y su libertad de cátedra. La asunción acrítica de modelos de coordinación fuerte puede conllevar la emergencia de una idea del docente que cada vez cobra más protagonismo: el profesorado proletarizado, desposeído de autonomía y cuyo papel se reduce a la mera transmisión del contenido y los métodos decididos desde la coordinación (Fernández-Enguita, 1990). Por tanto, estamos en un momento de transformación del modelo del docente «profesional» y la cultura organizativa que implica, pero no tenemos claro hacia qué avanzamos, estando presente el riesgo de la «proletarización» y la asunción de los modelos de cultura organizativa taylorista que conlleva (Noll, 2019).

Finalmente, no podemos obviar el último, pero no menos importante, factor determinante de los modelos de coordinación docente: las condiciones materiales. El viejo Marx diría en frase feliz: «No es la conciencia lo que determina la existencia, sino las condiciones materiales de existencia las que determinan la conciencia» (Marx y Engels, 2014[1932]: 21). ¿Cuáles son esas condiciones materiales en el caso de los departamentos universitarios?

En primer lugar, la carga docente y el tamaño del departamento parecen un condicionamiento evidente. No es lo mismo tener que coordinar a pocos docentes, que coordinar una asignatura que se imparte en diez grupos diferentes, los costes temporales y materiales son diferentes. En segundo lugar, la tasa de rotación del profesorado. Que exista una alta tasa de rotación del profesorado, con muchísimas entradas y salidas constantes de personal interino, despierta una acuciante necesidad de establecer modelos de fuerte coordinación para fijar unos criterios mínimos de calidad en la docencia (consensuar e imponer materiales, métodos, contenidos), y también, para ayudar a la incorporación del personal itinerante. Esta necesidad es más acuciante en el caso de universidades que, no sólo tienen una alta tasa de rotación, sino que además abusan de modalidades contractuales a tiempo parcial, en las cuales el profesorado interino tiene contratos de muy pocas horas y tienen que partirse con otros docentes la misma asignatura. En tercer lugar, y muy relacionado con lo anterior, la cantidad de profesorado «senior» también es muy relevante. El profesorado «senion», como su nombre indica, ha alcanzado un estatus académico y social, y tiende a operar, por derecho propio, bajo la lógica del docente «profesional», algo que puede desembocar en resistencias a la hora de establecer modelos de coordinación. A igualdad de ran- 
go ¿quién debe coordinar a quién? Esta situación puede generar conflictos que son resolubles mediante la horizontalidad y la negociación, pero alternativamente pueden obviarse al optar por un modelo de coordinación nula. En cuarto lugar, también creemos que es muy relevante el nivel de organización y movilización del estudiantado. Un estudiantado organizado, que protesta, denuncia y recurre ante los agravios comparativos en las formas de evaluación, contenidos, etc., empuja a establecer modelos de coordinación más cerrados.

\subsection{Elementos y características de la coordinación docente}

La labor de coordinación docente y el estilo de coordinación se concretan en una serie de elementos o ítems fundamentales: las guías docentes (y sus contenidos), el rol de la persona coordinadora, el grado de colaboración de los docentes y la flexibilidad del modelo. Es a partir de estos factores como definiremos nuestros tipos ideales de coordinación de asignaturas.

En primer lugar, las guías docentes son sin duda el principal elemento material en el que un modelo de coordinación se manifiesta. Las guías establecen competencias a adquirir por el alumnado, pero, sobre todo, establecen instrumentos y métodos de evaluación, contenidos y materiales. El mayor o menor grado de especificación y concreción de la guía en todos sus apartados, nos sitúa en el modelo de coordinación por el que se opta.

En segundo lugar, el rol de la persona coordinadora es otra característica fundamental de todo modelo de coordinación docente. Si bien sus competencias en términos normativos suelen estar más o menos definidas, en la práctica su rol varía fundamentalmente por la cultura organizativa dominante y por los condicionamientos materiales que afectan al departamento. Por ejemplo, en culturas organizativas basadas en el ideal del docente "profesional» el rol de la figura coordinadora será menos pronunciado. En situaciones con una alta rotación de personal y con un alumnado muy movilizado, la coordinación puede llegar a ser más relevante, asumiendo incluso funciones directivas o de supervisión de la labor docente.

En tercer lugar, el nivel o grado de colaboración efectiva es otro elemento definitorio relevante. El nivel de comunicación, interacción o intercambio de experiencias, materiales, etc., de los docentes refleja el mayor o menor peso de un modelo de coordinación. Aunque parte de este factor depende de las personas implicadas, la cultura organizativa tiene también un peso clave en el grado de colaboración que se espera del profesorado.

La flexibilidad del modelo es la última característica que identificamos. Podemos entender la flexibilidad como la capacidad de adaptación a diferentes situaciones sobrevenidas que tiene el modelo, en tres aspectos:

- Adaptación al docente. Entendiendo el grado en el que el modelo permite adaptar al docente la asignatura a sus conocimientos y aptitudes.

- Adaptación al estudiante. Entendida como el grado en el que el modelo de coordinación permite adaptarse a las distintas circunstancias que pueden aparecer en el aula: alumnado con necesidades especiales, eventual escasa matriculación y otras demandas del estudiantado. 
- Adaptación a la contingencia. La situación desatada por la COVID-19 ha permitido comprobar la capacidad del modelo de coordinación de adaptarse a situaciones excepcionales. Durante el último año se han producido idas y venidas constantes a un modelo híbrido, que no termina de ser ni presencial ni online. Pero esta capacidad puede ser empleada también ante otras contingencias.

\section{Modelos de coordinación docente en la enseñanza de la sociología en Andalucía} A través de la vivencia y experiencia propia en cuatro universidades andaluzas (la Universidad de Granada, la Universidad de Huelva, la Universidad Pablo de Olavide y la Universidad de Sevilla), se han construido dos modelos de coordinación de asignaturas. Estas dos formas, con carácter típico-ideal (y que, por tanto, no reflejan un estilo de coordinación concreto encontrado en alguna de las universidades), se han definido como los extremos de un continuo que va desde la «autonomía total» hasta la «coordinación total», dentro del cual es posible clasificar los modelos concretos realmente existentes. Para su construcción se ha partido de una reflexión colectiva autoetnográfica de la experiencia docente de las personas firmantes de este trabajo, las cuales, han hecho el esfuerzo de identificar cómo los factores determinantes y los ítems definitorios de los modelos de coordinación (descritos en el marco teórico) se materializaban en las distintas experiencias docentes que han tenido a lo largo de sus carreras en las distintas universidades. A partir de este ejercicio de compilación y síntesis, el autor principal realizó una primera clasificación de las mismas que posteriormente pasó a un proceso de debate y construcción colectiva al contrastarlo con las experiencias del resto de coautores. La riqueza del proceso llevado a cabo radica en la diversidad de posiciones desde las que parte cada participante, recogida a grandes rasgos en la tabla II.

Tabla II. Posiciones de partida de los autores para la autoetnografía

\begin{tabular}{|c|c|c|c|}
\hline & INVESTIGADOR 1 & INVESTIGADORA 2 & INVESTIGADOR 3 \\
\hline $\begin{array}{l}\text { Periodo de experiencia } \\
\text { docente }\end{array}$ & Curso $2015 / 2016$ y de 2018/2019 en adelante & Curso 2019/2020 y curso 2020/2021 & Curso 2006-2007 en adelante \\
\hline $\begin{array}{l}\text { Experiencia docente } \\
\text { (horas totales) }\end{array}$ & 842,4 & 120 & 2.385 \\
\hline Figuras contractuales & Contratado con cargo a proyecto, PSI & Becaria FPU & Becario FPU, PSI, Ayudante, PAD, PCD Interino, PCD \\
\hline $\begin{array}{l}\text { Modalidades contractuales } \\
\text { que ha tenido }\end{array}$ & Tiempo parcial y tiempo completo & Tiempo completo & Tiempo completo \\
\hline $\begin{array}{l}\text { Universidades andaluzas } \\
\text { en las que ha trabajado }\end{array}$ & UHU, UPO, US, UGR & UGR & UGR, US \\
\hline $\begin{array}{l}\text { Estancias en universidades } \\
\text { extranjeras }\end{array}$ & No & Sí & Sí \\
\hline $\begin{array}{l}\text { Experiencia en coordinación } \\
\text { de asignaturas }\end{array}$ & No & No & Sí \\
\hline
\end{tabular}

Fuente: elaboración propia.

Los resultados de esta investigación y reflexión colectiva se estructuran en dos partes. En primer lugar, se definen de manera detallada los dos tipos ideales de modelos de coordinación docente atendiendo a los ítems definitorios básicos antes descritos. En segundo lugar, se plantea una reflexión sobre las ventajas e inconvenientes de ambos modelos, a fin de hacer una valoración que ayude a mejorar los modelos de coordinación realmente existentes. 


\subsection{Coordinación total vs Autonomía total. Dos modelos típico-ideales de coordinación docente}

El primer modelo es el de autonomía total, un modelo que de manera ideal se correspondería con una estructura normativa indefinida (sin detallar funciones ni fines de la coordinación), con una cultura organizativa estructurada en torno a la idea del docente «profesional» y con unas condiciones materiales caracterizadas por un escaso número de asignaturas y personal docente a coordinar, una baja tasa de rotación del profesorado, un alto número de profesorado "senior" y un estudiantado menos activo. Este modelo de autonomía total se caracteriza por varias cuestiones.

En primer lugar, por unas guías docentes muy abiertas y generales, que dejan un margen de libertad muy grande al docente para adaptarla a sus intereses y a la realidad del aula. Este grado de indefinición de las guías docentes se manifiesta en el grado de especificación de los contenidos, los instrumentos de evaluación y los materiales. Por su parte, los contenidos (temario) son muy generales, tratando de escapar de temas cerrados y concretos y haciendo mención a grandes bloques y grandes conceptos. Por ejemplo: en una asignatura de Sociología General, este modelo concretaría módulos temáticos del tipo: 1) Introducción; 2) Sociedad; 3) Cultura; 4) Desigualdad; e incluso daría alguna indicación de que estos bloques pueden cambiar de orden si la persona encargada de la asignatura lo considera. Respecto a los instrumentos de evaluación, estos están totalmente indefinidos. En este modelo de autonomía se tienden a establecer unas normas generales, por ejemplo, estableciendo el porcentaje de la nota final que representan las prácticas y la teoría, pero sin definir instrumentos de ningún tipo mediante los cuales serán evaluadas, dando gran libertad al docente para planificar la evaluación. Por último, respecto a los materiales, estos son también muy generales. Las guías en este modelo suelen incluir algunas indicaciones sobre cuál puede ser la bibliografía de la asignatura, pero conteniendo una gran diversidad y heterogeneidad que permite enfocar la asignatura de miles de maneras distintas. Esto favorece que los materiales sean completamente personalizables y personalizados por el profesorado y, en la mayoría de los casos, este material no se comparte, dada la cultura organizativa dominante.

En segundo lugar, la figura de coordinación es simplemente inexistente. No obstante, si consideramos que este tipo ideal trata de definir un contexto universitario en el que los SGC han impuesto cierto entramado normativo que impone una figura de coordinación, la existencia y el papel de la misma es meramente nominativa, es decir, existe, pero su papel se reduce a eso, a existir, estando vacía de funciones reales.

En tercer lugar, respecto al nivel de colaboración de los docentes, dada la cultura organizativa dominante y las condiciones materiales (que se caracterizarían por el alto porcentaje de profesorado «senion» y un escaso número de docentes a coordinar), la colaboración es de carácter voluntario y usualmente muy baja, reduciéndose a colaboraciones esporádicas basadas en afinidades personales y relaciones informales.

Por último, como se deduce de todo lo dicho, la flexibilidad, entendida como capacidad de adaptación del modelo y como margen de libertad para adaptarlo que tiene el profesorado, es altísima en algunos sentidos, pero no así en otros. En lo que respecta a la capacidad de adapta- 
ción a la especialidad y competencia del docente, la flexibilidad es total. Dada la indefinición y ausencia formal de control, el profesorado puede transformar la asignatura, incorporando temas de su especialidad profesional o investigadora. Por ejemplo, en el caso de que el docente tenga una especialización fuertemente cuantitativa, podría plantear las prácticas de su asignatura de Sociología General, al manejo de un programa de análisis cuantitativo como SPSS o STATA, de igual manera que podría enfocarlas al debate y análisis político, en el caso en el que fuera especialista en la materia. También la capacidad de adaptación al estudiantado es muy alta. El docente tiene capacidad para readaptar la asignatura a las necesidades sobrevenidas. Por ejemplo, si de repente se encuentra con una clase con muy baja matriculación, el docente podrá ofrecer un modelo de evaluación más continua y personalizada, con otros instrumentos de evaluación como, por ejemplo, más actividades prácticas y trabajos, suprimiendo los exámenes. No obstante, la capacidad de adaptación a la contingencia pandémica es menor. La principal ventaja de este modelo, que es la autonomía en el ejercicio docente, se torna en este tipo de circunstancias en su principal inconveniente, el individualismo. El personal docente, aislado ahora no solo en términos culturales sino también materiales (encerrado en su casa, confinado), debe ser autodidacta, y son conocidos los costes que supone la curva de aprendizaje al modelo online, especialmente sin apoyo externo.

Frente a este modelo, tenemos su contrario, el modelo de coordinación total, un modelo caracterizado por una estructura normativa que define de manera clara y escrupulosa todas las funciones y metas de la coordinación; por una cultura organizativa estructurada en torno a la colaboración forzosa y al ideal del «docente proletarizado», que debe transmitir unos conocimientos estandarizados mediante unas técnicas dadas, lo que nos acerca al taylorismo educativo. Las condiciones materiales están definidas por la precariedad del profesorado, el escaso número de personal «senior», el alto grado de movilización del estudiantado y la necesidad de coordinación de un número relativamente elevado de docentes que imparten la misma materia. Veamos cómo se concreta este modelo en los ítems que estamos utilizando.

En primer lugar, respecto a las guías docentes, este modelo se caracteriza por unas guías docentes cerradas y muy bien definidas en todos sus aspectos. Respecto a los contenidos, tienen un temario claro y muy bien definido, hasta un grado extremo. Si el modelo anterior se caracterizaba por definir los temas en torno a grandes conceptos, este se caracteriza por todo lo contrario, definir el temario en torno a temas concretos y formulados en frases auto-explicativas más extensas. Por ejemplo, un temario de Sociología General, bajo este modelo, podría estructurarse de la siguiente manera: 1) Introducción a la Sociología como ciencia social. 1.1) Definición de Sociología según Macionis. 1.2) Los orígenes de la Sociología. 1.3) Primeras teorizaciones de lo social: Saint-Simon, Comte, Durkheim, Weber y Marx. 1.4) Paradigmas en Sociología: Funcionalismo y Conflicto.

En lo concerniente a los instrumentos de evaluación, estos también se encuentran estandarizados, no sólo limitándose a expresar qué porcentaje de la nota corresponde a teoría y prácticas, sino especificando cómo se evaluarán cada uno de estos apartados, indicando qué instrumento evaluará la teoría y cómo será, e incluso cuántas preguntas tendrá (por ejemplo: examen tipo test de 40 preguntas y 4 opciones de respuesta, dos preguntas mal restan una bien), y también 
concretando cuántas prácticas serán, cuánto vale cada una y en qué consistirán. En cuanto a los materiales, frente a la indefinición y generalidad del modelo de autonomía, en este modelo aparece la figura del «dossier». El mencionado «dossien» no sería otra cosa que un compendio en el que se contienen todos los materiales que el profesor debe impartir, casi en su literalidad, tanto para la parte teórica como para la parte práctica. Este «dossier» es compartido por todo el profesorado que imparte la misma materia y está especificado en la propia guía docente.

En segundo lugar, la figura de la coordinación de asignaturas es central en este modelo, hasta tal punto que podríamos afirmar que es el pilar fundamental que estructura el mismo. La persona coordinadora no sólo es la encargada formal de organizar el desarrollo general de la asignatura, sino que asume una serie de funciones que transforman su labor en un auténtico trabajo, en todo el sentido del término. La coordinadora es a la vez productora de contenido, coach, facilitadora, supervisora y mediadora. Sobre su figura recae la labor principal de elaboración de las guías docentes y los mencionados «dossiers», a la vez que tiene que dar la bienvenida al resto del profesorado, coordinarlo, reunirse para transmitirle los contenidos de la asignatura, resolver sus dudas y, muy importante, motivarles y convencerles de la pertinencia del modelo docente que ha diseñado. También hace labores de supervisión, garantizando que el profesorado imparta de manera estandarizada, y hasta sincrónica, el programa y materiales docentes contenidos en la guía. Por último, pero no menos importante, la persona coordinadora es también mediadora. Entre el alumnado y el resto del profesorado, en tanto que el alumnado puede dirigir sus quejas e inquietudes a la coordinadora, pero también entre el propio profesorado; en caso de discrepancias o conflictos la coordinación dirime.

En tercer lugar, el nivel de colaboración de los docentes es muy elevado, debido básicamente a que es un hecho obligado por la estructura misma del modelo. La coordinación contacta con el profesorado, a quien reúne de manera periódica, supervisa e invita a que establezcan relaciones entre sí para estar coordinados en tiempo en la impartición de contenidos y para que, en el margen que tienen, asuman criterios homogéneos. En una estructura normativa de colaboración forzosa y de cultura organizativa taylorista la coordinación se impone al docente de la misma manera que al obrero de la industria en cadena, la única diferencia es que no es el trabajo muerto reificado en la cadena de montaje a lo que se enfrenta a él/ella y le impone la cadencia de trabajo, sino un proyecto docente predefinido y controlado en su ejecución.

Por último, respecto a la flexibilidad, el modelo se caracteriza por una escasa capacidad de adaptación en casi todos los sentidos, pero no en todos. Respecto a la capacidad de adaptación al profesorado y al estudiantado el modelo es altamente inflexible. En un contexto marcado por un proyecto docente muy definido, con unos instrumentos de evaluación predeterminados y con un contenido materializado en la figura del «dossier», el margen de libertad del profesorado para adaptar el proyecto docente y las clases a aquellas cuestiones en las que es experto y a las demandas y necesidades del estudiantado desaparecen por completo. No obstante, respecto a la capacidad de adaptación a la contingencia pandémica, quizás sea el punto más fuerte de este modelo. Si bien por sus propias características el modelo resulta inflexible en los aspectos anteriores, respecto a la adaptación a un cambio repentino, como fue el paso de la enseñanza presencial a la online, el modelo parece tener grandes fortalezas. El hecho de que todo esté absolutamente 
claro y especificado, que la persona coordinadora tenga un papel central en la estructuración del modelo y que el profesorado esté muy acostumbrado a mantener una colaboración casi diaria, hace que el modelo resista mejor a los envites de la contingencia pandémica. Los instrumentos, los materiales y los métodos a asumir se construyen en común y se comparten de manera forzosa, esto hace al modelo más apropiado para dar respuesta a situaciones de cambio brusco. No obstante, hay que valorar que, lógicamente, la adaptación supone claramente un esfuerzo colectivo, pero, sobre todo, individual, de la propia persona coordinadora, en la cual recae fundamentalmente la responsabilidad de establecer el nuevo marco y también las dudas, inquietudes y problemas del resto de docentes de la asignatura.

Tabla III. Resumen de las características en las que se concretan los dos modelos típico-ideales de coordinación

\begin{tabular}{lll}
\hline & AUTONOMÍA TOTAL & COORDINACIÓN TOTAL \\
\hline Guías docentes & Abiertas y generales & Cerradas y muy definidas \\
\hline Temario & Bloques temáticos abiertos y generales & Bloques temáticos extremadamente cerrados \\
\hline Instrumentos de evaluación & Heterogéneos. A discreción del docente & Homogéneos y predefinidos \\
\hline Materiales & Individualizados y personales & Compartidos y homogéneos (dossier) \\
\hline Rol de la persona coordinadora & Meramente nominativo & Productor/a de contenido, coach, supervisor/a y mediador/a \\
\hline Colaboración entre docentes & Voluntaria (baja) & Obligatoria (alta) \\
\hline Capacidad adaptación al docente & Total & Nula \\
\hline Capacidad de adaptación al estudiante & Total & Nula \\
\hline Capacidad de adaptación a docencia virtual & Muy baja (individualizada) & Muy alta (construcción de instrumentos, medios y materiales compartidos) \\
\hline
\end{tabular}

Fuente: elaboración propia.

\subsection{Ventajas e inconvenientes de ambos modelos de coordinación docente}

La caracterización de los dos modelos extremos de coordinación docente que acabamos de exponer no tiene otro fin que invitar a reflexionar sobre las implicaciones que puede tener asumir unos modelos u otros. Si se observa la tabla III, se puede apreciar que las ventajas de un modelo son casi los inconvenientes del otro.

\section{Tabla IV. Resumen de las principales ventajas e inconvenientes de los dos modelos} típico-ideales de coordinación docente

\begin{tabular}{|c|c|c|}
\hline & AUTONOMÍA TOTAL & COORDINACIÓN TOTAL \\
\hline Ventajas & $\begin{array}{l}\text { - Plena autonomía del docente } \\
\text { - Fomento de la cualificación y el autodidactismo } \\
\text { - Capacidad de adaptación a las necesidades del grupo } \\
\text { - Capacidad de adaptación de contenidos al perfil del docente } \\
\text { - Flexibilidad en el diseño del calendario, materiales e instrumentos de evaluación } \\
\text { - Poca carga de trabajo para la figura coordinadora }\end{array}$ & $\begin{array}{l}\text { - Sentimiento de pertenencia al grupo } \\
\text { - Cooperación y respuesta coordinada a contingencias } \\
\text { - Construcción de redes intra-departamento } \\
\text { - Facilidad de inserción de nuevo profesorado (PSI) } \\
\text { - Evita y sanciona conductas desviadas } \\
\text { - Garantiza mismas condiciones y contenidos de evaluación (igualdad) } \\
\text { - Gran adaptación a la contingencia }\end{array}$ \\
\hline Inconvenientes & $\begin{array}{l}\text { - Aislamiento } \\
\text { - Baja colaboración } \\
\text { - Dificultad de construir redes intra-departamento } \\
\text { - Dificultad de inserción de nuevo profesorado (PSI) } \\
\text { - Dificultad sancionar conductas desviadas } \\
\text { - Agravios comparativos para el alumnado } \\
\text { - Baja adaptación a la contingencia }\end{array}$ & $\begin{array}{l}\text { - Autonomía muy limitada } \\
\text { - Descualificación parcial del profesorado (taylorismo) } \\
\text { - Poca o nula capacidad de adaptación a las necesidades del grupo } \\
\text { - Poca o nula capacidad de adaptación al perfil del docente } \\
\text { - Calendarios, materiales e instrumentos excesivamente rígidos } \\
\text { - Alta carga de trabajo para la figura coordinadora }\end{array}$ \\
\hline
\end{tabular}

Fuente: elaboración propia. 
En lo que respecta al modelo de autonomía total, sus principales ventajas radican principalmente en lo que define al propio modelo, es decir, en la gran autonomía y flexibilidad que un modelo indefinido permite al docente. En este modelo, el profesorado se plantea como profesional autónomo, con total capacidad para autodeterminarse los tiempos y métodos de trabajo, algo que no sólo reporta una sensación de bienestar personal, sino que es incluso un indicador de estatus social. Además, este margen de autonomía permite al docente una capacidad total para flexibilizar contenidos y adaptarlos a sus competencias y habilidades y a los requerimientos y demandas de su alumnado, permitiendo la construcción de modelos pedagógicos en los que, no sólo el personal docente se siente cómodo, sino que son altamente personalizables y adaptables a las circunstancias concretas del aula.

No obstante, la principal virtud de este modelo, la autonomía, puede convertirse en su principal defecto, derivando en ocasiones en aislamiento, soledad y anomia. La alta autonomía, tiene el coste de requerir una gran capacidad de trabajo autónomo, experiencia suficiente para desenvolverse en unas situaciones cambiantes y un alto grado de conocimiento de la materia a impartir, algo que no siempre ocurre. El profesorado más joven (especialmente el personal en formación predoctoral y que realiza sustituciones) pueden sentir este modelo como una amenaza. Especialmente compleja es la situación del personal interino, quien es llamado para incorporarse de un día para otro, y puede comenzar a dar clases desde el día siguiente a la firma del contrato, las más de las veces sin ningún apoyo para enfrentarse a asignaturas de las que normalmente no tiene un conocimiento experto.

Más allá de estos problemas de incorporación de nuevo profesorado, este modelo también supone un problema de cara a generar redes intradepartamentales. Al no establecerse una colaboración de cara a coordinar materias, es más difícil que el profesorado configure redes de colaboración, siquiera para tareas concretas, porque no existe la necesidad. Se refuerza así una cultura organizativa basada en la autonomía que puede repercutir negativamente en la adhesión del personal docente a la entidad.

Por último, un problema fundamental de este tipo de modelos es también la gran arbitrariedad y el inexistente control sobre la labor docente. Si bien al personal docente se le supone una gran capacidad de trabajo y vocación (algo no solo derivado de su formación, sino de los numerosos filtros que debe superar), la realidad es que un modelo basado en la autonomía total corre el riesgo de no tener mecanismos suficientes para detectar y corregir conductas irresponsables o fraudulentas por parte del personal docente, repercutiendo muy negativamente en el alumnado y en el prestigio del departamento como institución. Es más, sin irnos a estos casos extremos, este modelo supone otro problema que perjudica al alumnado, y que ocurre incluso cuando el personal docente hace bien su labor: los agravios comparativos. Un modelo que permite construir contenidos, metodologías e instrumentos dispares, según el criterio del docente, supone que una misma asignatura pueda reconvertirse y asumir una naturaleza muy diferente según el profesor/a que la imparta.

Gran parte de estos problemas, si no todos, son resueltos en el marco del modelo de coordinación total. En este modelo, donde contenidos y metodologías están muy definidos (materializados en el «dossier») y donde la figura coordinadora se transforma en supervisora, pero 
también en guía y apoyo, la incorporación de nuevo personal no supone ningún tipo de problema, en tanto que nada más entrar, la coordinadora da la bienvenida al docente, explicándole y facilitándole todo el plan pedagógico que está prediseñado. A la vez, la colaboración forzosa por la vía de la coordinación supone la creación de un sentimiento de pertenencia casi espontáneo y facilita de manera enorme la creación de redes de colaboración en el seno del departamento. El profesorado se suele conocer y está obligado a cooperar, por lo que la generación de redes informales que fomentan el trabajo en otras labores, como la investigación, es más probable. Además, al estar todas las docentes coordinadas (y supervisadas), es muy difícil que se den conductas desviadas que malogren el proceso de enseñanza-aprendizaje, y los agravios comparativos en lo que respecta a contenido y metodologías docentes desaparecen por completo. Por último, en el aspecto humano, el docente nunca siente esa sensación de soledad, en tanto que siempre cuenta con el apoyo de la coordinación y de sus compañeros/as, con los cuales comparte todo el material e inquietudes y problemas que puedan surgir. En este sentido, este sistema permite una gran capacidad de respuesta conjunta a las contingencias, como las ocurridas el pasado mes de marzo de 2020.

Sin embargo, en su virtud se encuentra su principal defecto. Como señalamos anteriormente, este modelo se configura como una suerte de taylorismo educativo, compartiendo exactamente los mismos problemas que Braverman (1974) y Marglin (1974) señalaban como problemas de la organización taylorista del trabajo. Ante un proyecto docente definido hasta el extremo, la labor del docente consiste básicamente en repetir y hacer lo que se le ordena desde el programa estipulado por la coordinación. El monopolio del conocimiento (y la cualificación) se concentra en el personal docente coordinador, mientras el resto del profesorado es reducido a meros impartidores semi-descualificados que, dado su rol, es fácilmente sustituible por cualquiera con unas dotes mínimas de memorización y con unas competencias oratorias básicas. Esto no sólo supone una descualificación parcial del docente y su proletarización, sino que además tiene consecuencias psicológicas. Al igual que el operario industrial de la cadena fordista, la persona docente en un modelo de coordinación total queda reducida a una mera pieza dentro de una maquinaria mucho más grande, cuya dimensión y finalidad puede llegar a ser incluso desconocida para sí misma, ejecutando un proyecto docente de manera acrítica sin comprenderlo, vaciando de sentido su labor, con las consecuencias que esto puede tener para su autoestima profesional o de cara a posibles conflictos en el seno del departamento.

Por último, cabe mencionar que las consecuencias negativas de este taylorismo educativo no sólo repercuten al docente, sino que también puede conllevar graves perjuicios sobre el alumnado. Al igual que el «hombre masa» de la etapa fordista (Alonso, 2005), el alumnado en este modelo recibe una formación estandarizada hasta el extremo, sin ningún tipo de posibilidad de adaptación a sus demandas y requerimientos.

\section{A modo de conclusión. La coordinación en un contexto de precariedad}

Garantizar una educación de calidad, versátil y adaptada a las demandas sociales y del mercado con unos recursos escasos y con un capital humano crecientemente precarizado ha sido, y es, el gran reto de la docencia universitaria en nuestro país. La creación de las figuras de coordinación docente parece alzarse como la forma de hacer frente a este reto. Sin embargo, pese a la proliferación de normativas internas 
sobre coordinación docente, la realidad de cada universidad, de cada departamento, dista de ser homogénea, presentándose varias formas de afrontarla. Para entender los distintos modelos de coordinación realmente existentes, en el presente trabajo se propone un continuo teórico que va desde los modelos de autonomía total, que hasta entonces habían dominado en la docencia universitaria y que se basan en una suerte de laissez faire docente fundamentado en la suposición de la competencia y excelencia del profesorado universitario; hasta los modelos de coordinación total, que no son otra cosa que la aplicación de los principios de la organización taylorista del trabajo a la práctica docente. En su definición típico-ideal, ambos modelos son problemáticos y difíciles de asumir en un contexto de precariedad como el actual. Por un lado, el modelo de autonomía puede suponer una carga de trabajo excesiva para los docentes precarios, especialmente para aquellos que tienen que asumir encargos docentes sobrevenidos sin un tiempo suficiente para su preparación. Suponer que un profesorado itinerante y mal remunerado puede y debe hacer la misma labor que un personal docente consolidado, estable y que tiene potestad para elegir lo que imparte es, no sólo una injusticia, sino un riesgo para la calidad de la docencia impartida. Por otro lado, el modelo de coordinación total puede ser una buena opción para ciertas situaciones de precariedad, pero a medio y largo plazo hace emerger los problemas propios de la organización taylorista del trabajo: descualificación, pérdida de motivación del docente, polarización en las cualificaciones de la plantilla y una excesiva estandarización de la docencia. Posiblemente cada departamento o centro requiera una combinación particular de ambos planteamientos. No obstante, la concreción de un modelo de coordinación debe partir de dos bases fundamentales. En primer lugar, de un análisis empírico de los determinantes y condicionamientos normativos, materiales y culturales de cada departamento y sus consecuencias en términos de calidad docente. En segundo lugar, de una valoración global de las ventajas e inconvenientes del modelo realmente existente. El carácter más teórico-reflexivo de este trabajo desaconseja hacer una apuesta normativa por unos u otros modelos. No obstante, podemos plantear algunas reflexiones sobre cómo asumir la labor de coordinación en un contexto de creciente precariedad. En primer lugar, la adopción de modelos de mayor coordinación o autonomía debería ser flexible y variable, adecuándose a las necesidades emergentes del departamento y de su plantilla. Un modelo de mayor coordinación, incluso de coordinación total, es recomendable en situaciones en las que los departamentos tienen que lidiar con una elevada rotación de personal, pero también para el personal docente en formación en general. Una mayor coordinación facilita la repentina incorporación del profesorado interino (especialmente con contratos de corta duración) o para dotar de herramientas y formar al personal docente de nueva incorporación. Sin embargo, conforme el profesorado se consolida, no sólo laboralmente (algo muy difícil en momentos de escasez de plazas), sino profesionalmente, demanda avanzar hacia una mayor autonomía, idealmente negociada y colaborativa, tendente a un modelo cooperativo progresivamente horizontal. En segundo lugar, la persona que coordina la asignatura tiene un rol fundamental. Se precisa una persona capaz de encabezar y fomentar la coordinación y colaboración y que a su vez haga de facilitadora ante eventuales problemas. Consolidar estas figuras requeriría su fomento a través de algún tipo de incentivo laboral, especialmente en los casos en los que el profesorado que se encarga de una materia es numeroso. La asignación de créditos por labores de coordinación podría ser una buena forma de reconocer la labor y evitar sobrecargar de trabajo a un profesorado ya saturado de tareas docentes e investigadoras. Por último, independientemente del modelo por el que se opte, es necesaria la construcción de mecanismos de participación y debate horizontal entre el profesorado de la asignatura independientemente del rango, evitando dinámicas directivas autoritarias. De esta manera, se podrían atender las necesidades y problemas de forma colectiva, y a la vez construir un espacio de cooperación e intercambio de experiencias, ideas 
y materiales. Para ello pueden servir iniciativas tan básicas como las reuniones periódicas, pero también prácticas de innovación docente que busquen redefinir de forma colaborativa el proceso de enseñanza de la asignatura. Más allá de estas recomendaciones y a modo de conclusión, creemos que el actual contexto de precariedad, devastador para las condiciones de vida y trabajo del profesorado, al menos ha puesto en evidencia la existencia de un modelo anterior de docencia tendente al solipsismo, donde era posible una práctica docente aislada e incuestionada y cada enseñante era una isla. La forzosa cooperación y el apoyo mutuo entre docentes es sin duda una de las pocas buenas noticias que podemos extraer del actual contexto precario. Pero esta constatación esconde una cara perversa: al mitigar los efectos negativos de la precarización, una coordinación más profunda y estructurada puede ser utilizada como ariete para profundizar en esta práctica, al tiempo que arroja sobre las espaldas del profesorado la responsabilidad de hacerla sostenible.

\section{Agradecimientos}

El presente trabajo es una versión elaborada de una sesión formativa que el autor principal impartió en el marco del equipo docente «Formar(se) en la contingencia: equipo docente de formación continua en metodologías multimodales de enseñanza-aprendizaje» (financiado en el marco de la X convocatoria de equipos docentes de formación de la Universidad de Granada), coordinado por la doctora Rosa María Soriano Miras y el doctor Mariano Sánchez Martínez. También queremos agradecer a los compañeros que colaboraron en la sesión, concretamente a Juan Miguel Valdera Gil y a Teresa Rodríguez Molina, los cuales nos aportaron ideas relevantes.

\section{Referencias bibliográficas}

Agulló-Fernández, Itzíar (2013): “Eternamente doctores ¿mérito o carga?”. Sociología del Trabajo, 78, 35-50.

Alonso, Luis Enrique (2005). La era del consumo. Madrid: Siglo XXI.

Bolarin-Martínez, María José (2016): "La coordinación docente en la universidad: análisis de la situación actual”. Educatio Siglo XXI, 34, 167-184. DOI: https://doi.org/10.6018/j/263861.

Bolarin-Martínez, María José y Moreno-Yus, M. Ángeles (2015): “La coordinación docente en la universidad: retos y problemas a partir de Bolonia. Profesorado". Revista de Currículum y Formación de Profesorado, $19(2), 319-332$.

Braverman, Harry (1974). Labor and Monopoly Capital. New York: Monthly Review press.

Burawoy, Michael (1989). El consentimiento en la producción: los cambios del proceso productivo en el capitalismo monopolista. Madrid: Ministerio de Trabajo y Seguridad Social.

Castillo, Juan José y Moré, Paloma (2016). Por una Sociología del trabajo académico. La precarización del trabajo de enseñar e investigar en la Universidad. Sociología del Trabajo, 88, 7-26.

Castillo-Olivares, José María.; Torrado, Esther y Escuela, Chaxiraxi (2020): “La Macdonalización del sistema universitario: un caso de explotación del profesorado precario en la Universidad de la Laguna y su crisis sindical". International Journal of New Education, 5, 118-132. DOI: https://doi.org/10.24310/ IJNE3.1.2020.8728. 
Conesa-Carpintero, Ester y González-Ramos, Ana M. (2018): "Neo-gerencialismo y auteridad en el contexto académico español y europeo. ¿Dos caras de la misma moneda?”. Politica y Sociedad, 55 (1), 257 282. DOI: http://dx.doi.org/10.5209/POSO.55883.

Díaz-Santiago, María José (2013): "Experiencia no académica: el profesorado asociado como actor doblemente precarizado". Sociología del Trabajo, 78, 51-71.

Díez-Gutiérrez, Enrique Javier (2009): "El Plan Bolonia: capitalismo académico superior”. El Viejo Topo, 256, 22-27.

Fernández-Enguita, Mariano (1990). La escuela a examen. Un análisis sociológico para educadores y otras personas interesadas. Madrid: Pirámide.

Fernández-González, Joseba (2014): "El estudio del impacto de los movimientos sociales: el caso del movimiento estudiantil anti-Bolonia”. Oxímora. Revista internacional de ética y política, 4, 1-22.

Gill, Rosalind (2014): “Academics, Cultural Workers and Critical Labour Studies". Journal of Cultural Economy, 7 (1), 12-30. DOI: https://doi.org/10.1080/17530350.2013.861763.

León-Sancho, Iñigo (2008): "Retrospectiva de las movilizaciones universitarias frente a la Ley Orgánica Universitaria (2000 a 2002)" en Xavier Albizu-Landa, Joseba Fernández-González y Jon Bernat-Zubiri (coords.). Movimientos estudiantiles. Resistir, imaginar, crear en la Universidad: Asamblea de Ciencias Sociales por una Universidad crítica. San Sebastián: Gakoa.

Ley Orgánica 6/2001, de 21 de diciembre, de Universidades, Boletín Oficial del Estado, 307, pp. 4940049425, 16 enero 2017.

Ley Orgánica 4/2007, de 12 de abril, por la que se modifica la Ley Orgánica 6/2001, de 21 de diciembre, de Universidades, Boletín Oficial del Estado 89, pp. 16241-16260, 17 enero 2017.

Marglin, Stephen A. (1974): "What Do Bosses Do? The Origins and Functions of Hierarchy in Capitalist Production". Review of radical political economics, 6 (2), 60-112. DOI: https://doi.org/10.1177\%2F0486 61347400600206.

Marugán-Pinto, Begoña y Cruces-Aguilera, Jesús (2013): “Fragmentación y precariedad en la Universidad". Sociología del Trabajo, 78, 10-34.

Marx, Karl y Engels, Friedrich (2014 [1932]). La ideología alemana. Madrid: Akal.

Ministerio de Universidades y Ministerio de Educación y Formación Profesional. (2021a). Estadística de Gasto Público en Educación. Series Temporales. Disponible en: http://estadisticas.mecd.gob.es/EducaJaxiPx/Tabla.htm?path=/economicas/gasto/series-2019-dp//10/\&file=gasto14.px.

Ministerio de Universidades y Ministerio de Educación y Formación Profesional. (2021b). PDI por tipo y modalidad de la universidad, tipo de centro, sexo y régimen de dedicación. Disponible en: http://estadisticas. mecd.gob.es/EducaJaxiPx/Tabla.htm?path=/Universitaria/Personal/EPU19//10/\&file=PDI0104. px\&type $=$ pcaxis \&L $=0$. 
Noll, Henry (2019): “Es el taylorismo, estúpido! Sobre la nueva organización científica de la investigación y la docencia en la Universidad Española”. Sociología del Trabajo, 95, 1-18. DOI: https://doi. org/10.5209/stra.66436.

Ortiz-Oria, Vicente Manuel; Jenaro, Cristina; García-Meilán, Juan José; Zabiauz, Begoña; Mayor, M. A. y Arana, José María (2011). Carga de trabajo en el EEES: La necesidad de coordinación docente entre asignaturas [Comunicación oral]. X Jornadas de Redes de Investigación en Docencia Universitaria: diseño de buenas prácticas docentes en el contexto actual. Alicante: Universidad de Alicante.

Real Decreto 1393/2007, de 29 de octubre, por el que se establece la ordenación de las enseñanzas universitarias oficiales, publicado en BOE núm. 260, de 30/10/2007.

Real Decreto 861/2010, de 2 de julio, por el que se modifica el Real Decreto 1393/2007, de 29 de octubre, por el que se establece la ordenación de las enseñanzas universitarias oficiales, publicado en BOE núm. 161 de 3 julio 2010.

Ruiz Esteban, Cecilia y Torrego-Egido, Luis (2011): "Evaluación de experiencias de implantación de los Grados en Educación Superior”. Revista Electrónica interuniversitaria de formación del profesorado, 14 (4), 17 21. DOI: https://doi.org/10.6018/reifop.

Santos-Ortega, Antonio; Muñoz-Rodríguez, David y Poveda-Rosa, Ma Manuela (2015): "En cuerpo y alma. Intensificación y precariedad en las condiciones de trabajo del profesorado universitario". Arxius, 32, 13-44. http://roderic.uv.es/handle/10550/49026.

Saura, Geo y Bolívar, Antonio (2019): "Sujeto académico neoliberal: cuantificado, digitalizado y bibliometrificado". REICE. Revista Iberoamericana sobre Calidad, Eficacia y Cambio en la Educación, 17 (4), 9-26. DOI: https://doi.org/10.15366/reice2019.17.4.001.

Tomàs, M.; Armengol, C.; Borrell, N.; Castro, D.; Esteve, J.; Feixas, M.; Garín, J. y Marquès, P. (2001): "El cambio de cultura en las universidades del siglo XXI". Educar, 28, 147-162. DOI: https://doi. org/10.5565/rev/educar.399.

\section{Notas biográficas}

José Manuel Torrado es doctor en Sociología por la Universidad de Granada y profesor en el Departamento de Sociología, Trabajo Social y Salud Pública de la Universidad de Huelva. Sus intereses de investigación se centran fundamentalmente en la sociología urbana, la geografía humana y la demografía, particularmente en el estudio del comportamiento residencial, las áreas metropolitanas y la movilidad cotidiana. En tiempos más recientes ha diversificado sus líneas hacia campos como la sociología de la salud, del trabajo y de la educación.

Henar Baldán es graduada en Sociología y ha realizado el máster "Problemas Sociales. Dirección y gestión de programas sociales" en la Universidad de Granada. Actualmente está realizando su tesis doctoral en la misma universidad tras obtener un contrato predoctoral (Ley 14/2011 Fpu) en 2019. Sus intereses de investigación se centran fundamentalmente en la sociología urbana, más concretamente en la movili- 
dad y la segregación residencial de tipo socioeconómico, siendo su principal tema de interés el estudio de las comunidades residenciales en el área metropolitana de Granada.

Ricardo Duque-Calvache es doctor en Sociología por la Universidad de Granada y profesor en el Departamento de Sociología de dicha institución. Ha completado su formación gracias a varias estancias en diferentes Universidades nacionales e internacionales. En la actualidad es investigador principal de varios proyectos de investigación financiados en convocatorias competitivas, tales como: «Aproximación multimetodológica al comportamiento residencial y la vida cotidiana —MARBEL- (PID2020-119569GA-I00)» y «Movilidad, vivienda y comportamiento residencial en Andalucía —MOVICRA— (P20_00571)». Sus intereses de investigación se centran en la sociología urbana y la demografía, particularmente en el estudio de las áreas metropolitanas, el comportamiento residencial y los procesos de gentrificación, así como en las consecuencias sociales de la COVID-19. 